\title{
Chemotherapy-induced fatal hepatitis B virus reactivation in a small-cell lung cancer patient
}

\author{
LEI QIN $^{1}$, FANG WANG ${ }^{2}$, BING-WEN ZOU ${ }^{3}$ and ZHEN-YU DING ${ }^{3}$ \\ ${ }^{1}$ Division of Head and Neck Cancer, Cancer Center, State Key Laboratory of Biotherapy, West China Hospital, Sichuan University, \\ Chengdu, Sichuan 610041; ${ }^{2}$ Department of Radiation Oncology, Affiliated Hospital of Hebei University, Baoding, \\ Hebei 071000; ${ }^{3}$ Division of Thoracic Oncology, Cancer Center, State Key Laboratory of Biotherapy, \\ West China Hospital, Sichuan University, Chengdu, Sichuan 610041, P.R. China
}

Received April 18, 2016; Accepted June 27, 2016

DOI: $10.3892 / \operatorname{mco} .2016 .989$

\begin{abstract}
Hepatitis B virus (HBV) reactivation during chemotherapy is a major concern and is widely reported, particularly in association with hematological malignancies and lymphomas. While lung cancer ranks first in incidence and mortality worldwide, HBV reactivation has been largely overlooked in this disease. As regards small-cell lung cancer (SCLC), HBV reactivation has rarely been reported. We herein report the case of a hepatitis $B$ surface antigen-seropositive SCLC patient in whom HBV was reactivated during the course of chemotherapy, despite preemptive use of lamivudine. The patient developed fulminant viral hepatitis and succumbed to liver failure. The aim of this report was to highlight the major but overlooked issue of HBV reactivation in SCLC, and suggest that agents more potent than lamivudine may be more efficacious in high-risk patients.
\end{abstract}

\section{Introduction}

It is estimated that over one-third of the world's population has been infected with hepatitis B virus (HBV), and patients who are seropositive for hepatitis B surface antigen $\left(\mathrm{HBsAg}^{+}\right)$account for $12 \%$ of cancer patients receiving chemotherapy $(1,2)$. HBV reactivation during or after chemotherapy and subsequent liver function impairment is a major concern (3). HBV reactivation by chemotherapy is widely reported in the literature for hematological malignancies and lymphomas, and less for solid tumors (4). Lung cancer ranks first in incidence and mortality worldwide (5); however, HBV reactivation has been largely overlooked in this disease. Only one study previously reported

Correspondence to: Dr Zhen-Yu Ding, Division of Thoracic Oncology, Cancer Center, State Key Laboratory of Biotherapy, West China Hospital, Sichuan University, 37 GuoXue Lane, Chengdu, Sichuan 610041, P.R. China

E-mail: dingzhenyu@scu.edu.cn

Key words: lung cancer, chemotherapy, hepatitis B virus, lamivudine a reactivation rate of $\sim 19 \%$ among $\mathrm{HBsAg}^{+}$patients with advanced non-small-cell lung cancer (NSCLC) (6). However, as regards small-cell lung cancer (SCLC), HBV reactivation has rarely been reported.

We herein report the case of an $\mathrm{HBsAg}^{+}$SCLC patient with HBV reactivation during the course of chemotherapy, despite preemptive use of lamivudine. The patient developed fulminant viral hepatitis and succumbed to subsequent liver failure.

\section{Case report}

A 56-year-old man was admitted to the West China Hospital (Chengdu, China) in September, 2014 due to cough and blood in the sputum. The patient was diagnosed with SCLC of the right lung (stage T3N2M0). In the diagnostic work-up, the serological tests revealed positivity for $\mathrm{HBsAg}, \mathrm{HBeAg}$ and $\mathrm{HBcAb}$. The HBV DNA load was $1.1 \times 10^{5} \mathrm{IU} / \mathrm{ml}$. The patient had mildly elevated alanine aminotransferase (ALT) and aspartate aminotransferase (AST) levels [57 and 41 IU/1; upper limits of normal (ULN), 50 and 40 IU/1, respectively]. The biochemistry profile and complete blood count were otherwise normal. The family history revealed that the patient's mother and two brothers had died from liver disease.

The patient was prescribed preemptive lamivudine (100 mg once daily) initiated 1 week prior to chemotherapy, followed by combination chemotherapy (etoposide with carboplatin for 3 cycles and with cisplatin for 1 cycle); he then received radiotherapy to the thorax and mediastinum. Due to severe hemoptysis, myelosuppression and acute pancreatitis, the treatment was interrupted several times and was continued for a total of 6 months. During this time, the tumor enlarged, which could not be readily attributed to chemotherapy failure. The patient later received another 2 cycles of etoposide and cisplatin chemotherapy. The treatment timeline is depicted in Fig. 1.

The HBV DNA load dropped below the detection limit with the advent of lamivudine and remained low for $>8$ months. However, it increased to $1.0 \times 10^{5} \mathrm{IU} / \mathrm{ml}$ after the completion of thoracic radiotherapy. At this time point, an HBV genetic analysis was performed (Table I). Lamivudine resistance was not identified and the patient continued with 
lamivudine administration. The HBV DNA decreased to $6.0 \times 10^{3} \mathrm{IU} / \mathrm{ml}$ and increased to $1.6 \times 10^{5} \mathrm{IU} / \mathrm{ml}$ after the final 2 cycles of chemotherapy. The ALT and AST levels remained stable (under the detection limit) for the entire time.

After 3 months, tumor progression was detected (Fig. 2). The laboratory examination revealed a sharply elevated ALT (from 117 to 557 IU/l), AST (from 184 to $886 \mathrm{IU} / \mathrm{l}$ ), and total bilirubin levels [from 14.8 (ULN, $28.0 \mu \mathrm{mol} / 1$ ) to $98.5 \mu \mathrm{mol} / 1$ ], despite intensive use of hepatoprotectants (Essentiale, Gluthion and Transmetil). The HBV DNA was $>5 \times 10^{7} \mathrm{IU} / \mathrm{ml}$. The HBV mutation status was again analyzed (Table I) and this time it revealed a definitive emergence of lamivudine resistance. The antiviral agent was switched to entecavir $(0.5 \mathrm{mg}$ once a day). However, the patient developed fulminant hepatitis and his condition progressively deteriorated, with emerging signs of cholenzyme separation, hemostatic dysfunction, hypoalbuminemia, hyperammonemia, hepatic encephalopathy and hepatorenal syndrome. Eventually, the patient succumbed to the disease on August 25, 2015. Autopsy was refused.

\section{Discussion}

We herein report the case of a SCLC patient who developed HBV reactivation during chemotherapy, despite preemptive lamivudine treatment. The HBV DNA level rebounded after the initial drop, together with the appearance of lamivudine resistance mutations in the viral gene. In addition, the disease progressed under continuous lamivudine administration. This was accompanied by significant deterioration of hepatic function, and the patient eventually succumbed to the disease.

The definition of HBV reactivation varies in the literature, but it commonly refers to an ALT level $>2$ ULN in combination
Table I. The HBV genetic analysis.

\begin{tabular}{lcc}
\hline $\begin{array}{l}\text { Mutation } \\
\text { site }\end{array}$ & $\begin{array}{c}\text { Abundance } \\
\text { (test 1) } \%\end{array}$ & $\begin{array}{r}\text { Abundance } \\
\text { (test 2) } \%\end{array}$ \\
\hline S213T & 50 & 100 \\
Q215H & 50 & 70 \\
M204I & 0 & 100 \\
V214A & 0 & 30 \\
\hline
\end{tabular}

with either an abrupt increase in HBV replication of $1 \log _{10}$ or an absolute value $>2 \times 10^{4} \mathrm{IU} / \mathrm{ml}$ (7). In the majority of the cases, HBV reactivation occurred 1 week- 3 months post-chemotherapy (8). The patient's HBV DNA increased to $1.0 \times 10^{5} \mathrm{IU} / \mathrm{ml} 3$ months after the fourth cycle of chemotherapy. The time course was typical of chemotherapy-induced HBV reactivation. Furthermore, the virus genetic analysis revealed lamivudine resistance mutations. Considering the full course of HBV reactivation in a background of chemotherapy, it was reasonable to hypothesize that the chemotherapy was responsible for the HBV reactivation.

Chemotherapy-induced $\mathrm{HBV}$ reactivation has been extensively investigated. In retrospective series, the rate of HBV reactivation was as high as $80 \%$ in lymphomas or other hematological malignancies, particularly those treated with immunoinhibitory rituximab (3). The incidence of HBV reactivation in lung cancer had not been elucidated until recently, when a retrospective study reported a rate of $19 \%$ in NSCLC patients (6). However, SCLC patients have not been investigated, with no studies specific to SCLC patients reported to

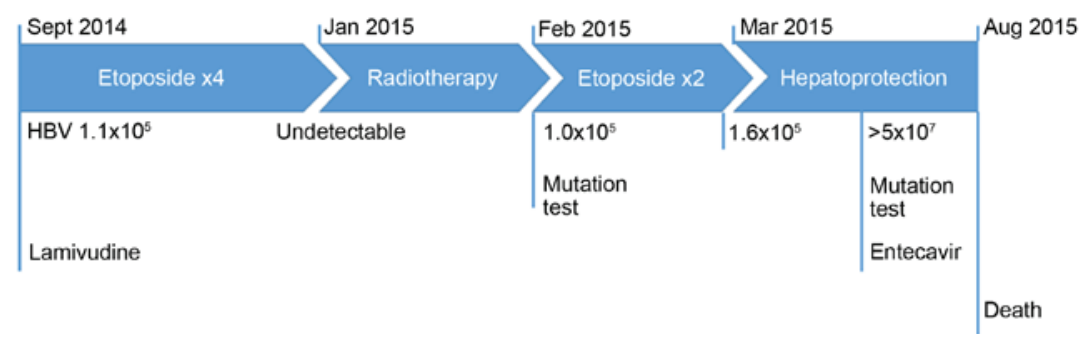

Figure 1. Summary of the treatment timeline of the patient. HBV, hepatitis B virus DNA load (IU/ml).

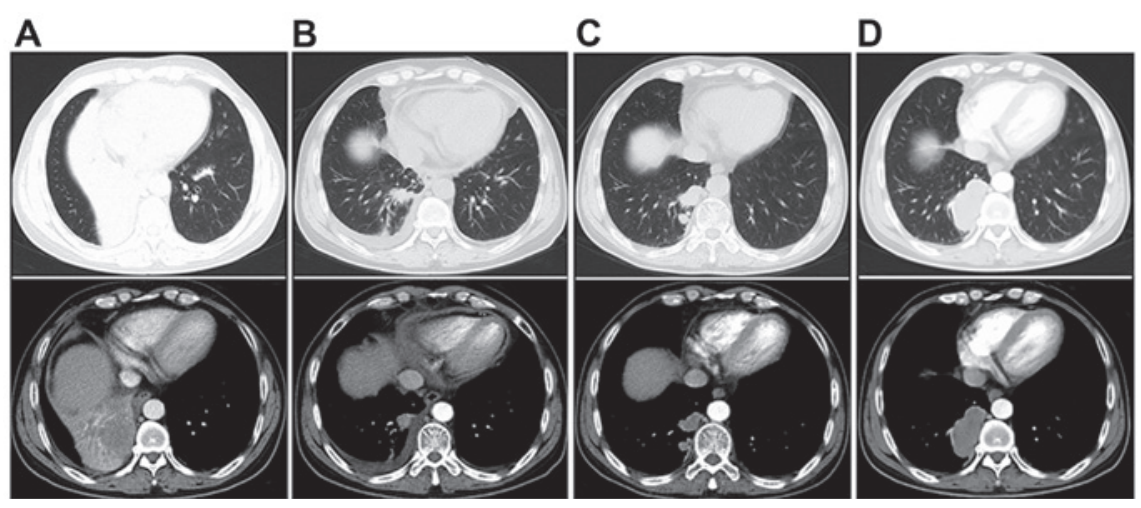

Figure 2. Tumor mass during the chemotherapy course. (A) Computed tomography scan revealed a mass in the lower lobe of the right lung. (B) The mass had significantly shrunk after 2 cycles of chemotherapy. The mass (C) marginally enlarged prior to the fifth cycle of chemotherapy and (D) significantly enlarged 1 month after cessation of chemotherapy. 
date. To the best of our knowledge, this is the only reported case of chemotherapy-induced fatal HBV reactivation in a SCLC patient.

The reasons underlying the lack of reports on HBV reactivation in SCLC patients remains largely unknown, although it may be due to the low incidence of this complication. Patients with hematological malignancies and lymphomas have the highest rates of HBV reactivation, mainly due to the intense chemotherapy and administration of immunoinhibitory monoclonal antibodies; in addition, such patients are already immunocompromised (3). Breast cancer patients also exhibited high rates of HBV reactivation, mainly attributed to the wide use of anthracyclines and corticosteroids. The HBV reactivation rates were lower in other cancer types. Another possibility is that SCLC has a dismal prognosis, and patients may become seriously ill before HBV reactivation is detected.

The reason underlying the poor prognosis is the evolution of the HBV gene, as evidenced by the serial monitoring of the lamivudine resistance gene. However, the patient's family history of liver disease must be taken into consideration, and the possibility of liver fragility cannot be ruled out. In addition, the patient had other comorbidities, such as severe hemoptysis, myelosuppression and acute pancreatitis. Thus, the fatal outcome may be attributed to a combination of all these factors.

Preemptive antiviral therapy is recommended for $\mathrm{HBsAg}^{+}$ cancer patients prior to the initiation of chemotherapy $(3,6,9)$. Accumulating data demonstrated that lamivudine reduced the incidence of HBV reactivation and the severity of hepatitis in these patients (10-13). The value of novel antiviral drugs, such as entecavir, telbuvidine, adefovir and tenofovir, is being evaluated. Recently, a trial compared the efficacy of lamivudine and entecavir in $\mathrm{HBsAg}^{+}$patients with diffuse large B-cell lymphoma undergoing rituximab treatment (14). Compared with lamivudine, entecavir significantly reduced the incidence of HBV reactivation and HBV-related hepatitis. It was reasonable to hypothesize that high-risk patients may benefit from the usage of entecavir rather than lamivudine.

In summary, this was a rare case of SCLC with fatal HBV reactivation during the chemotherapy course. This aim of this report was to highlight the major issue of $\mathrm{HBV}$ reactivation in SCLC, which is frequently overlooked, and suggest that agents more potent than lamivudine may be more efficacious for high-risk patients.

\section{Acknowledgements}

The present study was supported by the Natural Science Foundation of China (grant no. 81272684).

\section{References}

1. Ganem D and Prince AM: Hepatitis B virus infection-natural history and clinical consequences. N Engl J Med 350: 1118-1129, 2004.

2. Yeo W, Chan PK, Zhong S, Ho WM, Steinberg JL, Tam JS, Hui P, Leung NW, Zee B and Johnson PJ: Frequency of hepatitis B virus reactivation in cancer patients undergoing cytotoxic chemotherapy: A prospective study of 626 patients with identification of risk factors. J Med Virol 62: 299-307, 2000.

3. Mandala M, Fagiuoli S, Francisci D, Bruno R, Merelli B, Pasulo L, Tondini C, Labianca R and Roila F: Hepatitis B in immunosuppressed cancer patients: Pathogenesis, incidence and prophylaxis. Crit Rev Oncol Hematol 87: 12-27, 2013.

4. Yeo W and Johnson PJ: Diagnosis, prevention and management of hepatitis $\mathrm{B}$ virus reactivation during anticancer therapy. Hepatology 43: 209-220, 2006.

5. Siegel R, Naishadham D and Jemal A: Cancer statistics, 2013. CA Cancer J Clin 63: 11-30, 2013.

6. Lin GN, Peng JW, Xiao JJ, Liu DY and Xia ZJ: Hepatitis B virus reactivation in hepatitis $B$ surface antigen seropositive patients with metastatic non-small cell lung cancer receiving cytotoxic chemotherapy: The efficacy of preemptive lamivudine and identification of risk factors. Med Oncol 31: 119, 2014.

7. Liaw YF and Chu CM: Hepatitis B virus infection. Lancet 373: 582-592, 2009.

8. Hoofnagle JH, Doo E, Liang TJ, Fleischer R and Lok AS: Management of hepatitis B: Summary of a clinical research workshop. Hepatology 45: 1056-1075, 2007.

9. Lok AS and McMahon BJ: Chronic hepatitis B: Update 2009. Hepatology 50: 661-662, 2009.

10. Yeo W, Chan PK, Ho WM, Zee B, Lam KC, Lei KI, Chan AT, Mok TS, Lee JJ, Leung TW, et al: Lamivudine for the prevention of hepatitis B virus reactivation in hepatitis B s-antigen seropositive cancer patients undergoing cytotoxic chemotherapy. J Clin Oncol 22: 927-934, 2004.

11. Lau GK, Yiu HH, Fong DY, Cheng HC, Au WY, Lai LS, Cheung M, Zhang HY, Lie A, Ngan R and Liang R: Early is superior to deferred preemptive lamivudine therapy for hepatitis $B$ patients undergoing chemotherapy. Gastroenterology 125: 1742-1749, 2003.

12. Hsu C, Hsiung CA, Su IJ, Hwang WS, Wang MC, Lin SF, Lin TH, Hsiao $\mathrm{HH}$, Young JH, Chang MC, et al: A revisit of prophylactic lamivudine for chemotherapy-associated hepatitis B reactivation in non-Hodgkin's lymphoma: A randomized trial. Hepatology 47: 844-853, 2008

13. Long M, Jia W, Li S, Jin L, Wu J, Rao N, Feng H, Chen K, Deng $\mathrm{H}$, Liu F, et al: A single-center, prospective and randomized controlled study: Can the prophylactic use of lamivudine prevent hepatitis B virus reactivation in hepatitis B s-antigen seropositive breast cancer patients during chemotherapy? Breast Cancer Res Treat 127: 705-712, 2011.

14. Huang H, Li X, Zhu J, Ye S, Zhang H, Wang W, Wu X, Peng J, $\mathrm{Xu} \mathrm{B}$, Lin Y, et al: Entecavir vs lamivudine for prevention of hepatitis $B$ virus reactivation among patients with untreated diffuse large B-cell lymphoma receiving R-CHOP chemotherapy: A randomized clinical trial. JAMA 312: 2521-2530, 2014. 